\title{
Semantics and Evolution of "Che" in Anyue Dialect
}

\author{
Yanglu Zhou* \\ School of Arts, Southwest University, Chongqing 400715, China
}

*Corresponding author: Yanglu Zhou, z18723192864@163.com

\begin{abstract}
In Anyue dialect, the meaning of the word "che" is very rich. It can be used as a verb to express the meaning of "argument," "discussion," "tearing," "receiving," “cutting," "picking," “opening," "buying," and "pulling." It can also be used as an adjective to express the meaning of "immoral," "unreliable," and "naughty." This article describes the semantics and usage of "che" in Anyue dialect as well as the usage of special phrases with "che" in other areas of southwest Mandarin. Finally, this article analyzes the semantic evolution of "che" and constructs a semantic evolution diagram.
\end{abstract}

Keywords: Anyue dialect; "Che"; Semantic evolution

Publication date: September 2021; Online publication: September 30, 2021

\section{Role of enterprise practice implemented by vocational colleges in industrial parks}

Anyue dialect belongs to the Chengdu Chongqing area of the southwestern Mandarin. At present, the academic research on Anyue dialect mainly includes the following perspectives: first, the phonetic perspective, which is the phonetic characteristics of Anyue Daping dialect; second, the lexical perspective, which involves the research on " $\mathrm{X}$ dao" words; third, the grammatical perspective with the research on "V xia" format. However, these studies do not include "che" in Anyue dialect. In Anyue dialect, the word "che" is used as an adjective in addition to the same verb usage as in Mandarin. The frequency of its use is very high. Based on the spoken language of Anyue dialect, this article describes and analyzes the rich semantic usage of the term "che" in Anyue dialect, explores its meaning, and also describes its special usage in other dialects, hoping to enrich the research on "che" in Chinese dialects.

\section{Semantics and usage of "che"}

It can be seen that the term "che" is a common word for "pull," but it does not only mean "holding something by hand." According to Ciyuan, "che" also means tearing and meaningless conversation. In Anyue dialect, "che" does not only have the above meanings, but also other meanings and usages.

\subsection{Used as a verb}

In Anyue dialect, "che," as a verb, does not only have the meaning of "pull," but also "discuss," "argue," "buy," and "receive." It is usually used as a predicate in a sentence.

\subsubsection{Its meaning of "discuss," "argue," and "speak"}

"Che" in Anyue dialect can express the meaning of "discuss," "argue," and "say," which can be used alone or followed by phrases.

Example 1: Do not rush into something, and then only discuss about what to do (che yi xia). 
Example 2: Do you have to discuss over such a small matter for so long? (che hen jiu)

Example 3: Do not speak to him; it is not clear. (gen shui che)

Example 4: You have been gossiping all day. (che dong che xi)

Example 5: Do not lie to me. (che huang)

Example 6: Do not worry, we will discuss about it again. (che huir)

Example 7: The two of them had a dispute today. (che pi)

When "che" is used as a predicate in a sentence, it is used alone and should not be followed by components. However, when it is necessary to supplement the semantics of the sentence, the corresponding object or complement can be added after the word "che."

\subsubsection{Its meaning of "tear"}

In Anyue dialect, the meaning of "tear" is roughly the same as that in Mandarin. It is used as a predicate in a sentence, followed by an object or verb complement.

Example 8: Tear some paper for me. (che zi)

Example 9: Do not fight, your clothes will get torn. (che le)

Example 10: Xiaoming lost his temper and tore the notebook. (che ben zi)

\subsubsection{Its meaning of "receive"}

"Che" in Anyue dialect has the same meaning as in Mandarin. It is usually a fixed collocation. It is generally only seen in "receiving a marriage certificate" and "receiving a divorce certificate."

Example 11: Xiao Hong and Xiao Ming are going to get their marriage certificate today. (che zheng)

\subsubsection{Its meaning of "cut," "pick," and "open"}

In Anyue dialect, when "che" means "cutting," "picking," and "opening," it is usually used as a predicate followed by an object, but the specific meaning needs to be judged in terms of the context.

Example 12: Go and cut some cloth to make clothes. (che bu)

Example 13: Xiaoming went to the field to pick vegetables today. (che cai)

Example 14: Take off the banner. (che xia lai)

Example 15: After dinner, I had the bill invoiced for reimbursement. (che piao)

As can be seen from the above examples, when "che" ("pull") in the Anyue dialect means "cut," "pick," and "open," the subsequent nouns are often accompanied by the action of "tearing."

\subsubsection{Its meaning of "pull" and "hold"}

Example 16: Do not pull your hair every day. (che tou fa)

Example 17: Do not pull my clothes. (che yi fu)

Example 18: Hold on her or she will run away. (che dao)

\subsubsection{Fixed collocation phrases}

In Anyue dialect, "che" also has some fixed collocation phrases to express special meaning. For example, 
“snore," "scream," and "making trouble."

Example 19: He makes snoring sounds every night. (che pu han)

Example 20: Xiaoming likes to make weird sounds during class. (che guai jiao)

Example 21: Xiao Ming does not like to eat vegetables; he makes trouble every time he eats. (che guai)

\section{2. "Che" as an adjective}

When "che" in Anyue dialect is used as an adjective, it expresses the meaning of "immoral," "talkable," "naughty," and "unreliable." However, the specific meaning must be explained in context.

Example 22: He is not easy to teach; he is very naughty. (che de hen)

Example 23: He is so unreasonable that no one dares to persuade him. (na me che)

Example 24: You are so talkable that no one can speak to you. (zui ba che de han)

Example 25: You are so ridiculous. (hao che you)

Example 26: He fools around all day. (gao che de han)

\section{Special phrase with "che" in other areas of southwest Mandarin}

The word "che" exists in a large area in modern Chinese dialects. The word "che" in modern Chinese is mainly distributed in southwest Mandarin areas (Hubei, Sichuan, Chongqing, Yunnan, and Guizhou), Hunan areas (Hunan), Fujian areas (Hainan and Fujian), Jiaoliao Mandarin areas (Shandong), Lanyin Mandarin areas (Ningxia), and other dialect areas close to southwest Mandarin. In these distribution areas, some of its meanings are different from those in Anyue dialect. This section discusses the special collocation of the word "che" in Chengdu and Chongqing dialects as well as the difference with its use in Anyue dialect.

In Chengdu dialect, "che" also has some fixed collocation phrases with other different meanings.

Example 27: Who is joking with you? Someone is really looking for you. (che ba zi)

Example 28: Do not listen to him talking nonsense. (che ba zi)

Example 29: He has nothing to do; he is chatting with others over there. (che ba zi)

The meaning of "che" in the above examples represents "joking," "boasting," and "chatting," which have been changed from the meaning of "discussion" or "debate." However, these phrases are not used in the Anyue dialect.

In Mianyang and Zitong dialects, "che" and "che bai" are used, which indicate "talking nonsense."

Example 30: Stop talking nonsense and sleep. (che bai tan jing)

Example 31: Why do you start talking nonsense at this time? (luan che bai)

In Chongqing dialect, "che" also has a fixed collocation phrase "che ya ba jin," which also means "fighting" or "arguing." This phrase is not common in Anyue dialect.

Example 32: I have a lot of things to do; I do not have time to argue with you. (che ya ba jin)

\section{Semantic evolution of "che"}

"Che" has the meaning of "pull," "tear," and "gossiping" in modern Chinese dictionaries, whereas in Anyue 
dialect, it has very rich meaning, including "discussing," "tear," "receive," and "picking." This section discusses the semantics of "che" from the perspective of diachrony and analyzes the process of its semantic evolution.

The original meaning of "che" is "tearing." According to the Chinese corpus of Peking University, the word "che" was first used in the Five Dynasties.

Example 33: He was pulled by a pinch over his head, caught his clothes and pulled his arms. (che bi) (a selected work from Dunhuang Bianwen)

From the Five Dynasties to the Song Dynasty, "che" came about to have the meaning of "pulling" and "dragging." They are associated with the action of "tearing." The meaning of "tearing" is expanded to directly express hand actions, which is to tear things apart by hand.

Example 34: At the end of his speech, he pulled the emperor's cloak with his hand, looked at the gates of heaven and pushed it. (che zhu) (Xuanhe Legacy of the Great Song Dynasty)

Example 35: Cui Ning was also dragged to be a ghost with her parents. (che qu) (Jade Grinding Guanyin)

During the Yuan Dynasty, "che" had the meaning of "lifting" and "holding." From a horizontal action of "pulling" and "dragging" by hand, it expanded to a vertical action. However, this meaning is no longer used in the Anyue dialect.

Example 36: He held his Longquan sword in his hand. (che zai) (Du Liucui in the Yuan Dynasty)

In the Ming and Qing Dynasties, "che" began to have an extended meaning of "pulling up," such as pulling seedlings and hair, which also involves hand movements.

Example 37: Under the peach blossom, the phoenix pulled its feathers. (sheng che) (Zheng Xuzhou's Grand Unveiling Post - Fragrance of Royal Clothes in the Ming Dynasty)

During the Ming and Qing Dynasties, "che" developed an oral action meaning, relating to speech. It mostly refers to rambling conversations, gossips, and talking nonsense. This meaning gradually changed from the meaning of dispute action between two people to the speech action between them. However, when "che" expresses this meaning, it cannot be used as a word alone.

Example 38: Do not talk nonsense; do you know who I am? (che dan) (Chapter 2 of Xingshi Marriage Biography)

Example 39: He lied as he has never heard of the mountain. (che huang) (Chapter 19 of A Dream of Red Mansions)

Example 40: It is nonsense to think about being an official. (che dan) (San Gui Ji by Ji Zhenlun of Ming Dynasty)

Example 41: Nonsense! He is Nezha. I want to see him too. (che sao) (Chapter 7 of A Dream of Red Mansions)

As can be seen from the above examples, "che" has completed the transfer from the hands to the mouth when it expresses conversational meaning. The subsequent objects show that "che" does not fully have 
verbal meaning and cannot be used alone. Later, it gradually evolved into an independent word and no longer depended on other verbal verbs (phrases). When it is not used with the verbal noun object, "che" produces a verbal meaning and develops into a verb to express the meaning of "speaking improperly."

Example 42: After speaking improperly about the past and the present, Zijinying's face flushed. (che wan, che gu jin) (Ruan Zhangjing's Zhang River Water)

The semantic evolution process of "che" is summarized as follows: "crack" and "open" were the original meanings but often, the term was used to explain actions so its meaning extended to "tear"; the action of "tearing" required the completion of the action by hands; thus, it was further refined to the meaning of "pulling" and "dragging," indicating a horizontal action as well as "lifting," "holding," and "pulling up," indicating a vertical action; since most of the hand actions involved two people, "che" gradually evolved from hand actions between two people to verbal actions and verbal verbs between two people; a verbal verb with no verbal meaning and depended on other verbal verbs or noun objects was developed; finally, the oral verb, "che" with oral meaning, was developed and eventually, the oral verb has been used to express the meaning of "debate" and "discussion." In Anyue dialect, "che" is also used as adjectives, expressing "joking," "powerful," "naughty," and "unreliable." These meanings require a specific context to judge the meaning of "che" in the sentence. However, these meanings generally are derogatory in nature. It can be generally used to describe a person's performance or habits which are inconsistent with common sense. In order to explain this meaning, based on speculations, there are two possibilities: one is to expand from the meaning of "immoral" and "meaningless conversation" to describe a person's immorality; the other comes from the meaning of "pulling up," indicating a separate from the original environment and moving into a new environment. For example, "pulling radishes" and "pulling feathers" deviate from the original track. In Anyue dialect, it has evolved into the meaning of "naughty" and "immoral" to describe people who are separated from the environment and different from the people around them. The semantic evolution of "che" is shown in Figure 1.

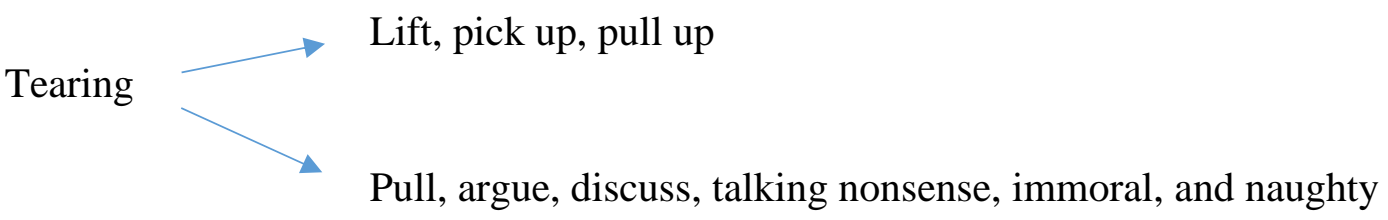

Figure 1. Semantic evolution of "che"

\section{Conclusion}

The word "che" is frequently used in Anyue dialect and has rich semantics. It can be used not only as a verb to express the meaning of "argument," "discussion," "tearing," "receiving," "cutting," "picking," "opening," "buying," and "pulling," but also as an adjective to express the meaning of being "immoral," "unreliable," "naughty," and "a joke." In Anyue dialect, there are also some fixed collocation phrases such as "che guai jiao," "che guai," and "che pu han," which express fixed meanings: deliberately making fun, deliberately making trouble, and snoring, respectively. "Che" can also be used as an adjective evolved from the meaning of "pull up" or "improper conversation." "Che" is widely distributed in modern Chinese dialect areas, including southwest Mandarin, Jiaoliao Mandarin, and Lanyin Mandarin. In Chengdu and Chongqing dialects, there are some special phrases with the word "che." In Chengdu dialect, "che ba zi" expresses the meaning of joking, boasting, and chatting. In Chongqing dialect, "che ya ba jin" indicates fighting or arguing. The semantic evolution process extends from "split" to "tear". As the action of "tear" is often 
accompanied by hand actions, it extended to "pull," "lift," and "take." As they are generally actions between two people, it further extended to verbal debate and argument between two people.

\section{Disclosure statement}

The author declares that there is no conflict of interest.

\section{References}

[1] Zhang Y, Zhang Q, Deng Y, 2001, A Study of Chengdu Dialect Grammar, Bashu Publishing House, Sichuan.

[2] Wu F, 2019, Semantic Evolution and Lexical Evolution, Research on Ancient Chinese.

[3] Fudan University, Kyoto Foreign Studies University, Japan, 1999, Dictionary of Chinese dialects, Zhonghua Book Company, Beijing.

[4] Editorial Committee of Chinese Dictionary, 2010, Chinese Dictionary, Chongwen Publishing House, Sichuan Dictionary Publishing House, Wuhan, Chengdu.

[5] Deng Y, Zhang Y, 2010, Vocabulary Research of Sichuan Dialect, China Social Sciences Press.

[6] Dong Z, 2009, Transfer from Hand Action to Mouth Action in the Evolution of Word Meaning, Chinese.

[7] Zhu Q, 2014, Research on Chinese "Ti" Verbal Verbs - From Hand Action Meaning to Verbal Meaning, Shanghai Normal University. 